\title{
Uso de la Plataforma Smile para la Elaboración de Conceptos en Estudiantes en Repitencia en un Programa de Ingeniería
}

\author{
Marlin A. Aarón \\ Fac. De Ingenierías. Programa de Ingeniería de Sistemas, Universidad de La Guajira Riohacha, La Guajira, \\ Colombia (e-mail: maaron@uniguajira.edu.co)
}

Recibido Feb. 28, 2018; Aceptado May. 14, 2018; Versión final Jul. 24, 2018, Publicado Abr. 2019

\begin{abstract}
Resumen
Se pretende explicitar el proceso implementado durante la enseñanza y el aprendizaje a través de una didáctica caracterizada por la indagación, el acompañamiento permanente y el diálogo participativo con estudiantes de primer semestre del Programa de Ingeniería de Sistemas, repitentes de la Asignatura Introducción a la Ingeniería de Sistemas. Se desea que los estudiantes enfrenten la metodología aprendizaje combinado (blended learning), apoyada en la Plataforma Smile y lograr la generación de conceptos durante la construcción de preguntas esenciales derivadas de estos contenidos. La experiencia se enmarca metodológica-mente desde la Investigación-Acción-Participación, un estudio cuali-cuantitativo en el que se hace uso de la estadística para reconocer los cambios en la elaboración de las preguntas. Se logra ejercitar en los estudiantes habilidades de pensamiento de orden superior desde gestionar información, analizar, pensar con información, y la construcción de preguntas y respuestas usando Smile. El mayor nivel de pensamiento crítico se logró en la evaluación de las preguntas elaboradas por sus compañeros.
\end{abstract}

Palabras clave: plataforma Smile; estrategia de indagación; construcción de preguntas; taxonomía

\section{Use of the Smile Platform for the Development of Concepts in Students in Repeat in an Engineering Program}

\begin{abstract}
The aim of this work is to explicitly describe the process implemented during teaching and learning through a didactic characterized by investigation, the permanent accompaniment and the participative dialogue with students of the first semester of the Systems Engineering Program, repeaters of the Subject Introduction to Engineering of Systems. The idea is approaching students to experience a blended learning methodology supported by the Smile Platform and achieve the generation of concepts during the construction of essential questions derived from these contents. The experience is methodologically framed by the Action Participation Research, a qualitative-quantitative study in which statistics are used to recognize the changes in the elaboration of questions. Students are able to exercise higher order thinking skills from managing of information, analyzing and thinking with information and constructing questions and answers using Smile. The highest level of critical thinking was achieved in the evaluation of the questions elaborated by classmates.
\end{abstract}

Keywords: Smile platform; inquiry strategy; higher order skills; construction of questions; taxonomy. 


\section{INTRODUCCIÓN}

El uso de una estructura para desarrollar un acto de aula, da cuenta de planificar la clase teniendo en cuenta en contexto, todo lo que se necesita pedagógica, didáctica y tecnológicamente. Al respecto lo que se considera es que desde la evidencia que muestran algunos estudiantes, dentro y fuera del aula, de atender y construir sus propias formas para el aprender, se concibe un escenario en el aula que sirva para posibilitar la forma como ellos se organizan para su proceso de aprendizaje. Acorde a Hederich (2010), muchos investigadores coinciden en afirmar que los estudiantes que se dedican a estudiar de manera consiente, construyen sus propias "herramientas" cognitivas y motivacionales para conseguir un aprendizaje eficaz, tal como lo indican Vargas y Martinez citando a Winne. (2010). De acuerdo con Paris y Byrnes (1989) este tipo de estudiantes tienen alta motivación hacia el aprendizaje y en consecuencia, se formulan metas concretas, planifican actividades para el logro de esas metas, monitorean el desempeño durante la ejecución de tales actividades, se evalúan continuamente de acuerdo con las metas y criterios fijados y finalmente, valoran el producto del proceso de aprendizaje. Persisten ante dificultades en el desarrollo de las tareas y de forma continua mejoran y adaptan sus estrategias de aprendizaje en diferentes contextos y situaciones. Schunk y Zimmerman (1994) definen esto como un aprendizaje autorregulado, en el que se construye un proceso a través del cual los estudiantes activan y mantienen cogniciones, conductas y afectos orientados al logro de sus metas de aprendizaje, lo que también consideran Torrano y Gonzales (2004). Por tanto, se puede afirmar que los estudiantes que regulan su aprendizaje son capaces de construir conocimiento de forma significativa y orientarse intrínsecamente para el logro de metas de aprendizaje.

Frente a este escenario en el cual es posible encontrar estudiantes que son capaces de generar sus propios estados de aprendizaje, desarrollando acciones que son valoradas por los docentes y por ellos mismos, que traen resultados positivos, es interesante mirar, cuando se encuentra frente a estudiantes, que desde la mirada de ellos y del docente, realizan acciones para aprender y no se logra el conocimiento. Lo que se evidencia son resultados de manera superficial y además que estas acciones no ayudan a su desarrollo personal ni su aprendizaje. Durante el desarrollo de la docencia de la asignatura de Introducción a la Ingeniería de Sistemas, primer semestre de 2015, se presentó una situación que generó alarmas pedagógicas, académicas y sociales, que fueron objeto de análisis. Un grupo de 45 estudiantes caracterizados por asistir regular y puntualmente a clases, que permanecían durante todo el tiempo, cumplían con la realización de tareas, más no con el propósito de las mismas, al ser valorados teniendo en cuenta los trabajos en clases y actividades, más del $60 \%$ de ellos no superó el mínimo básico que permitía evidenciar el conocimiento del tema ni competencias. Los exámenes escritos eran realizados por los estudiantes, con iguales resultados que las tareas. La docente frente a los resultados iníciales de tareas y primer parcial, modificó la didáctica, por un ejercicio permanente de explicación detallada de las orientaciones que se entregaban en las sesiones de clase, al encontrar que buena parte de los estudiantes, no comprendían lo que se les solicitaba y además les costaba el aprendizaje del concepto, el análisis y la explicación. Los conceptos se presentaban durante dos sesiones, una inicial, y la siguiente sesión presencial, como repaso. Los estudiantes participaban en la presentación de los temas, en el desarrollo de la clase, a través de una estrategia didáctica denominada, "el estudiante en el rol del profesor", en el que un estudiante, orientaba el inicio de la sesión de clases con una temática previamente definida.

Todo esto, dentro de un clima de disposición por parte de los estudiantes y su participación en todas las actividades. Era contradictoria su disposición, asistencia, cumplimiento en la realización de todas las tareas y sus resultados en los aprendizajes y construcción de conocimiento. Al final del curso, los estudiantes en su mayoría no superaron los objetivos de aprendizaje, no evidenciaron comprensión temática y baja competencia analítica. Los estudiantes manifestaron querer repetir la asignatura antes que habilitarla, - una opción final para superarla- con la misma docente, lo que se convertía en un reto pedagógico.Para la docente el reto académico fue concebir y poner en marcha en contexto (Gonzalvez, 2016; Huapaya et al., 2005) una estrategia pedagógica que diese cuenta de una didáctica activa que involucrase de manera permanente a los estudiantes, que superaran sus dificultades y lograr la apropiación de los contenidos temáticos de la asignatura de Introducción a la Ingeniería de Sistemas. El objetivo central respondía a construir una didáctica para la elaboración de conceptos y favorecer la comprensión temática, competencias de análisis y capacidades de pensamiento crítico usando la plataforma Smile.

La Stanford Mobile Inquiry-based Learning Environment, (Kim, 2008; Acosta y Reátegui, 2014), SMILE es una plataforma diseñada como entorno de aprendizaje basado en la indagación (Suárez, et al, 2018) (Lee, 2012). Esta herramienta tecnológica unida a una didáctica activa pretende favorecer la acción pedagógica. La plataforma Smile, de la Universidad de Stanford, entorno virtual, al que se accede desde internet y posibilita la construcción de preguntas por parte de los estudiantes que son vistas por todos los compañeros de curso y pueden ser respondidas. Los estudiantes construyen las preguntas y también las respuestas, lo que demanda conocimiento temático, coherencia en la escritura, sentido y comprensión. Un estudio desarrollado por Hsu y Kim (2016) con el fin de explorar maneras en que los maestros pueden usar Smile 
para apoyar aprendizaje en los estudiantes, probó la tecnología con 26 maestros de pregrado. Utilizaron Smile para provocar y utilizar pensamiento de orden superior en sus estudiantes en el proceso de aprendizaje. Evaluaron preguntas generadas por maestros y estudiantes de Kinder-12 grado (USA), reconociendo la satisfacción percibida y los desafíos del uso de Smile para mejorar las prácticas de cuestionamiento generadas por los estudiantes en el aula.

El desarrollo de la didáctica esta soportada en varios elementos que se integran metodológicamente. Uno de ellos es la elaboración de conceptos, para el que se espera que una acción planificada en el aula, pueda operar de manera positiva en él estudiante y lograr una apropiación de la acción que se pretende, teniendo como base lo que manifiesta Reeve (2003), cuando dice que ofrecer un incentivo interesante, representa una estrategia de motivación extrínseca (Rodríguez y Remesal, 2007; Maquilón y Hernández 2011), en este caso, es el uso de la Plataforma Smile (Buckner y Kim, 2014).En ese caso, tiene sentido diseñar un andamiaje, (Raes et al., 2012) tal como manifiesta Bruner (Bruner, 1978), que es una aproximación didáctica para desarrollar la capacidad de los estudiantes para realizar acciones que si puedan ayudarle a aprender. Parte del concepto de zona próxima de Vigotsky (Arceo, 2007; Marcano, 2006; Vigotsky, 1978). El andamiaje que se dispone se convierte en la didáctica que desarrolla el docente para ayudar al estudiante. Se busca de manera controlada identificar y generar acciones que le ayuden en su aprendizaje. $Y$ va poco a poco entregando tareas al estudiante que si puede desarrollar y se queda con las que no, y poco a poco genera una estructura en la que las actividades entregadas al estudiante no superan sus capacidades, y con esto se pretende que el estudiante vaya construyendo sus propias capacidades autoreguladoras. En la medida que esto ocurre, el estudiante puede concentrarse en el dominio de los aspectos relevantes de una estrategia o habilidad con cierta rapidez a través de la retroalimentación y apoyo social, en el momento en que ello sea requerido (Azevedo y Hadwin, 2005).

Según Hederich (2010) el diseño de andamiajes, como facilitadores para el desarrollo de habilidades en el proceso de regulación del aprendizaje, se ha vuelto un reto para quienes trabajan las tecnologías de la información aplicadas a la educación debido a que su potencial puede ser usado en el diseño de escenarios computacionales que sirvan de puente entre la autorregulación propiamente dicha y el aprendizaje externamente regulado en ambientes hipermedia y Web (Azevedo, et al., 2004). En este sentido, el reto que se gesta, está dado en que el docente define un andamiaje de enseñanza soportado en la elaboración de conceptos, como una estrategia para posibilitar que los estudiantes identifiquen "que es", "comprendan el concepto" y lo "expliquen". Lo que es posible hacerlo desde el uso de la plataforma tecnológica Smile, que está soportada en la construcción de preguntas que buscan el pensamiento crítico. Desde esta perspectiva, el docente se apoya en una mediación tecnológica para montar el andamiaje, tal como lo manifiestan Badia y García (2006), la metodología se caracteriza por una didáctica en la que el docente precisará de varios tipos de herramientas tecnológicas para proporcionar ayudas educativas útiles a los estudiantes, que deberán quedar integradas en una denominada aula virtual (Barberà et al., 2004). En nuestro caso, la plataforma tecnológica Smile, se unirá al aula virtual que se tiene para la asignatura de Introducción a la Ingeniería de Sistemas que está montada en Akumaja, que es una LMS soportada bajo Moodle donde está alojada el aula virtual de la asignatura, para posibilitar el blended learning (Pina, 2004; Turpo 2010) lo que es de dominio por parte de los estudiantes. Ellos parten de un escenario conocido, el aula de clases, la plataforma Akumaja, para llegar a usar una nueva mediación que es la plataforma Smile, a la que se llega a través de un enlace dispuesto en el aula virtual, y usarla para apropiar conceptos. Se pretende que ellos recuperen su conocimiento tácito (Nonaka, 2009) y lo vuelvan explicito (Aaron et all., 2014).

En este caso, el andamiaje que se usa es explicito, y tal como lo identifican Hadwin y Winne (2001), los andamiajes explícitos se refieren a "la inclusión intencional y evidente de herramientas dentro del ambiente computacional, las cuales son usadas por los aprendices a la hora de afrontar la tarea y requieren de su esfuerzo a la hora de alcanzar el dominio de conocimiento". Este será un nuevo esfuerzo por parte de los estudiantes, el aprendizaje de la plataforma Smile, pero con la intención de que ella sea mediadora para el reforzamiento de conceptos, tratando de alcanzar un aprendizaje eficaz por parte de los estudiantes. Se buscó la elaboración de conceptos usando un andamiaje explícito a través de la plataforma Smile y con este aprendizaje soportado en el "¿qué es?”, “¿cómo es?”, y “¿como se explica que...?”, conocimiento comprensión - análisis, construir una base de conocimiento que permita desarrollar cognitivamente a los estudiantes y propiciar en ellos una dinámica de aprendizaje, de tal forma que las acciones que ellos autónomamente desarrollen produzcan resultados positivos.

Los estudiantes elaboran preguntas temáticas que dan cuenta de sus aprendizajes, desde una taxonomía, Conocer, Comprender y Analizar CCA, retomada de Blomm, citado por Widiana et al. (2016), para pasar de habilidades de orden inferior a habilidades de orden superior. Las preguntas de conocimiento, dan cuenta del primer nivel, que?, cuantas? cuándo? preguntas fácticas; las de comprensión, por qué? para qué?, cuál?, explican procesos y las de tercer nivel, las de análisis, cómo?, combinaciones de cómo y cuál? requieren de los estudiantes pensamiento profundo y crítico. De allí la importancia de provocar en ellos mayor nivel de tipos 
de preguntas para fortalecer la conceptualización, la comprensión temática y la elaboración de las respuestas que según Tornero et al., (2015) son un medio para elevar el desarrollo cognitivo de los estudiantes. El contenido temático se desarrolla alrededor de Ciencia, Ingeniería, Sistemas, Informática y Computación, características de los sistemas, Problemas y algoritmos, Cibernética organizacional, Dinámica de sistemas, Teoría General de Sistemas, Organización de datos, Ingeniería de software e Integridad del software, Inteligencia Artificial, Telecomunicaciones,. Minería de Datos, Gestión de la Información y del Conocimiento, Computación Gráfica y la base formativa de la Ingeniería de Sistemas. Una didáctica activa dentro de una estrategia de indagación (Frías, 2011), que buscó integrar el aprendizaje con la habilidad para construir preguntas, usando como mediación una plataforma tecnológica, denominada Smile.

Se espera que al final, los estudiantes avancen en competencias de orden superior y transiten hacia el análisis y pensar con información. Aquí la plataforma tecnológica, sobre la cual se construyen preguntas temáticas, se usa como escenario para elaborar conceptos, y se convierte en un medio para lograr un fin. Esto unido a lo manifestado por Seol y otros, que el uso de la plataforma Smile demostró el desarrollo de competencias de conocimiento, comprensión y análisis a partir de la elaboración de preguntas sobre un tema en estudiantes de nivel primaria (Seol et al., 2011). El uso de esta plataforma se convirtió en el andamiaje que se dispuso y sobre el que se buscó alcanzar resultados que ayudasen a los estudiantes en repitencia, superar sus debilidades. La pregunta problema estaba centrada en ¿Cómo acercar a los estudiantes a la comprensión de los contenidos de la asignatura de Introducción a la Ingeniería de Sistemas y superar las dificultades y debilidades que presentaban en su condición de estudiantes repitentes, usando la plataforma Smile?

\section{METODOLOGÍA}

Se pone en marcha una metodología cuali-cuantitativa para reconocer los aprendizajes de los estudiantes. Se gesta desde la Investigación - Acción participación y se hace uso de la estadística para reconocer los cambios en la construcción de preguntas en cuanto a la cantidad de preguntas elaboradas y para analizar los resultados de los exámenes y las notas obtenidas. Se hace análisis de contenido, para identificar los tipos de preguntas que los estudiantes elaboran. La observación participante junto con el dialogo participativo en el aula de clases fue permanente. Se observaron los cambios en las formas como los estudiantes reaccionaban a las actividades propuestas para la inserción de la mediación tecnológica que es Smile. Es un estudio de caso, fenomenológico, que analizó la situación de 20 estudiantes en estado de repitencia. Los estudiantes provenían de dos cursos iniciales, el $80 \%$ con la misma docente. La didáctica estuvo centrada en la integración de la pedagogía y la tecnología para facilitar la construcción de conceptos desde la indagación, soportada en una taxonomía que diera cuenta del CCA, Conocer, Comprender y Analizar, usando la plataforma Smile, con énfasis en la apropiación de conceptos, la generación de competencias digitales, y capacidades de pensamiento crítico. Estos conceptos, correspondían a los núcleos temáticos de la asignatura.

Etapas y Proyección. Primera Etapa: Análisis Situacional: Caracterización de los aprendizajes y vivencias adquiridas durante el primer curso en el cual se desarrolló la asignatura. Descripción sintética de la experiencia. Se obtuvo información a partir de un cuestionario cerrado, con preguntas sobre conocimiento previo, acceso a internet, gustos por usar PC y razones de haber perdido la asignatura. Segunda Etapa: Diseño e implementación participativa de la didáctica a seguir para la construcción de preguntas y la elaboración de conceptos, Articulación de las sesiones presenciales con sesiones en la plataforma Smile y blended learning. Se usó la observación participante y cuestionario cerrado después de cada sesión para reconocer aprendizajes: se pide autoevaluación de sus preguntas, evaluación de las de sus compañeros, dificultad para elaborarlas, fuente para la elaboración de preguntas y respuestas, gusto y acceso a la plataforma Smile. Se hizo análisis de contenidos para las preguntas elaboradas. Tercera Etapa: Evaluación formativa sobre la didáctica establecida para desarrollar la taxonomía orientadora del proceso de enseñanza y aprendizaje de los contenidos temáticos de la asignatura. Diálogos participativos con los estudiantes para analizar las actividades, sesiones formativas realizadas usando la estrategia de indagación y análisis de los resultados de cada una de las evaluaciones parciales. Cuarta Etapa: Socialización de los significados que se otorgan a los aprendizajes adquiridos través de la sistematización de experiencias durante la comprensión temática de contenidos, y la construcción de preguntas como base para la elaboración de conceptos.

Se crearon 5 sesiones de trabajo dentro de la plataforma Smile, que servían como entorno para el acceso individual de los estudiantes para crear sus propias preguntas, así como para el entorno colectivo, en el que se permitió acceder a las preguntas de los compañeros y responderlas. Los estudiantes podían elaborar en cada sesión, la cantidad de preguntas que quisiesen, siempre que correspondieran a los temas ya vistos en clase previa o la actual. La información sobre las preguntas que se elaboraban en cada sesión fue objeto de análisis, alrededor de Conocimiento, Comprensión y Análisis.

Se generó una ruta de enseñanza y aprendizaje, un andamiaje pedagógico que sirvió para posibilitar logros académicos y el aprendizaje de contenidos y competencias en la asignatura de Introducción a la Ingeniería 
de Sistemas. Puede servir como modelo o caso de estudio para grupos similares en la que los estudiantes enfrenten situaciones que puedan tener relación con esto. Su puesta en marcha ayudó a resolver un problema real, para el que la Universidad dispuso una respuesta. La metodología que se puso en marcha, puede ser probada para la especial situación del contexto de estudio, pero también puede ser usada en otro contexto, en el que apropiarla, deba servir para la apropiación de conceptos, su comprensión y análisis.

\section{RESULTADOS Y DISCUSIÓN}

Los estudiantes reconocen que la mayor razón por haber perdido la asignatura fue no haber estudiado con el tiempo suficiente, no comprender los temas y creer que no era muy importante la asignatura. El uso de la plataforma Smile generó un entorno de aprendizaje activo e interactivo al posibilitar que los estudiantes construyeran preguntas y respuestas para temas de clases, así como responder las preguntas de sus compañeros de aula. Ellos se vieron enfrentados no solo al reto de pensar un tema, construir una pregunta para ese tema, construir las posibles respuestas, de las cuales una era la acertada y responder las preguntas de sus compañeros. Además evaluar las preguntas de sus compañeros y las propias, para identificar si estaban bien construidas. Se logró la interacción de los estudiantes para responder las preguntas. Se posibilitó un ambiente de indagación en el aula de clases. Los estudiantes construyeron preguntas que se relacionan en la tabla No. 1 que están categorizadas acorde a los interrogantes. La segunda y la última sesión fue donde hubo el mayor número de preguntas elaboradas. Ambas fueron previas al desarrollo de evaluaciones. Los mayores porcentajes de construcción fueron para las preguntas cuyo interrogante es el “¿qué?", preguntas de conocimiento.

En las tabla 2 se categorizan las preguntas por conocimiento, comprensión y análisis. Se identifica que las preguntas que más elaboraron estaban direccionadas hacia la definición de conceptos y comprensión de los temas. Las que menos, preguntas analíticas. Fue aumentando en cada sesión en la que combinan mayormente el uso de interrogantes cual y cómo. Nunca hubo una sesión en la que todos construyesen preguntas de este tipo. Al analizar las sesiones de trabajo se encontró que estas preguntas fueron construidas por 5 estudiantes, los que en algunos casos, escribieron más de una pregunta.

Tabla 1: Preguntas por interrogantes elaboradas por los estudiantes

\begin{tabular}{|l|l|l|l|l|l|l|l|l|l|l|}
\hline $\begin{array}{l}\text { Sesiones de } \\
\text { construcción } \\
\text { de preguntas }\end{array}$ & $\begin{array}{l}\text { No de } \\
\text { preguntas } \\
\text { construidas }\end{array}$ & $\begin{array}{l}\text { ¿Qué } \\
\text { es? }\end{array}$ & ¿Cuál? & $\begin{array}{l}\text { ¿Para } \\
\text { qué? }\end{array}$ & ¿Cómo? & $\begin{array}{l}\text { ¿Por } \\
\text { qué? }\end{array}$ & ¿Quiénes? & ¿Cuántas? & ¿Cuándo? & $\begin{array}{l}\text { Preguntas } \\
\text { que } \\
\text { combinan } \\
\text { Cuály } \\
\text { Cómo }\end{array}$ \\
\hline No. $126 / 08$ & 38 & 18 & 7 & 2 & 0 & 2 & 5 & 2 & 2 & 0 \\
\hline No. 2. $9 / 09$ & 93 & 73 & 10 & 4 & 2 & 0 & 0 & 0 & 0 & 4 \\
\hline No. 3. $23 / 09$ & 25 & 11 & 4 & 4 & 1 & 0 & 0 & 0 & 0 & 5 \\
\hline No. 4. $21 / 10$ & 39 & 13 & 3 & 2 & 10 & 2 & 0 & 0 & 0 & 9 \\
\hline No. 5. $11 / 11$ & 87 & 39 & 19 & 2 & 7 & 8 & 0 & 0 & 0 & 12 \\
\hline
\end{tabular}

Tabla 2: Construcción de preguntas categorizadas por CCA

\begin{tabular}{|l|c|c|c|c|}
\hline $\begin{array}{c}\text { Sesiones de } \\
\text { construcción de } \\
\text { preguntas }\end{array}$ & $\begin{array}{c}\text { No de } \\
\text { preguntas } \\
\text { construidas }\end{array}$ & $\begin{array}{c}\text { CONOCIMIENTO } \\
\text { que, quienes, cuantas, } \\
\text { cuando }\end{array}$ & $\begin{array}{c}\text { COMPRENSIÓN } \\
\text { cual, para que, } \\
\text { como, por que }\end{array}$ & $\begin{array}{c}\text { ANALISIS Preguntas } \\
\text { que combinan Cuály } \\
\text { Cómo. }\end{array}$ \\
\hline No. 1 26/08 & 38 & 27 & 11 & 0 \\
\hline No. 2. $9 / 09$ & 93 & 73 & 16 & 4 \\
\hline No. 3. $23 / 09$ & 25 & 11 & 9 & 5 \\
\hline No. 4. $21 / 10$ & 39 & 13 & 17 & 9 \\
\hline No. 5. $11 / 11$ & 87 & 39 & 36 & 12 \\
\hline
\end{tabular}

Los resultados de los exámenes (3) dos parciales y uno final, mostró un cambio en los estudiantes, en cuanto a la apropiación de conceptos, las competencias digitales y el sentido crítico de cómo aprender. Una de las mayores dificultades de los estudiantes era la comprensión de las instrucciones, se observó en los exámenes, que las actividades propuestas fueron analizadas asertivamente. La construcción de preguntas y de respuestas usando la plataforma Smile mejoró su concentración. La disposición de los estudiantes fue un factor importante para el desarrollo de un nuevo proceso de enseñanza. Su reconocimiento frente al hecho de estar repitiendo la asignatura, les instaba a superar las debilidades que ellos consideraban habían tenido. La figura 1 presenta los resultados de los estudiantes del primer parcial en el que se evidencia logros en sus aprendizajes, acordes con el conocimiento que se esperaba fueran apropiando. 


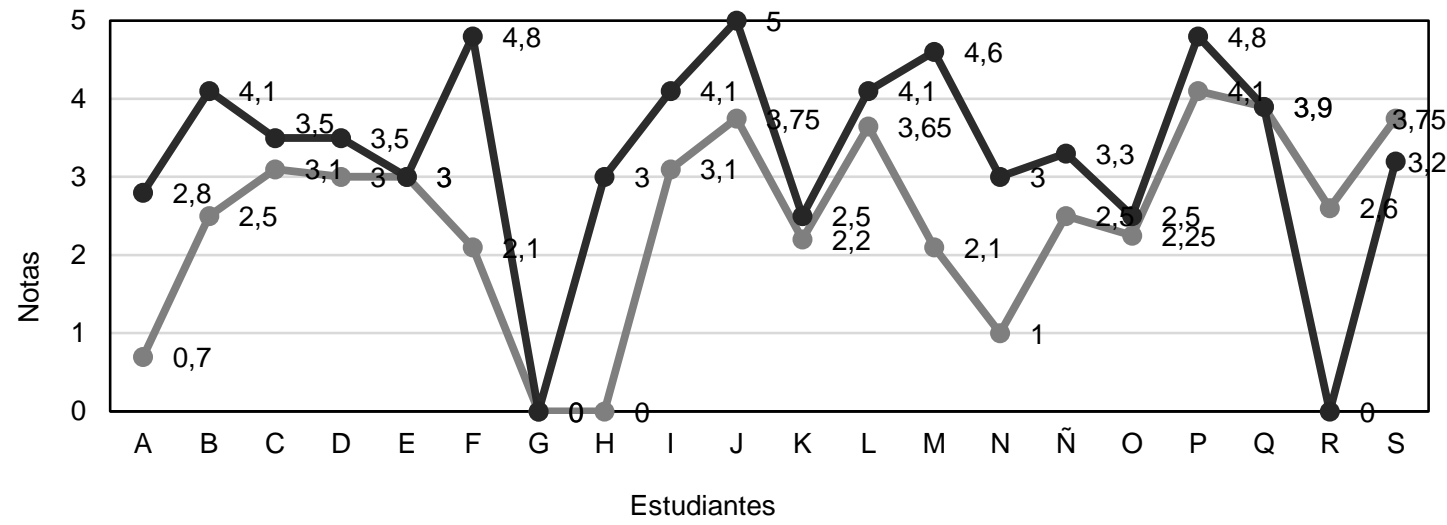

-Primer Parcial 2015-1 —Primer Parcial 2015-2 SMILE

Fig. 1: Resultados exámenes Primer Parcial I y II Sem 2015

La fig. 2 presenta los resultados de los estudiantes en el segundo parcial; los estudiantes demuestran en su evaluación que han ido apropiando conocimiento.

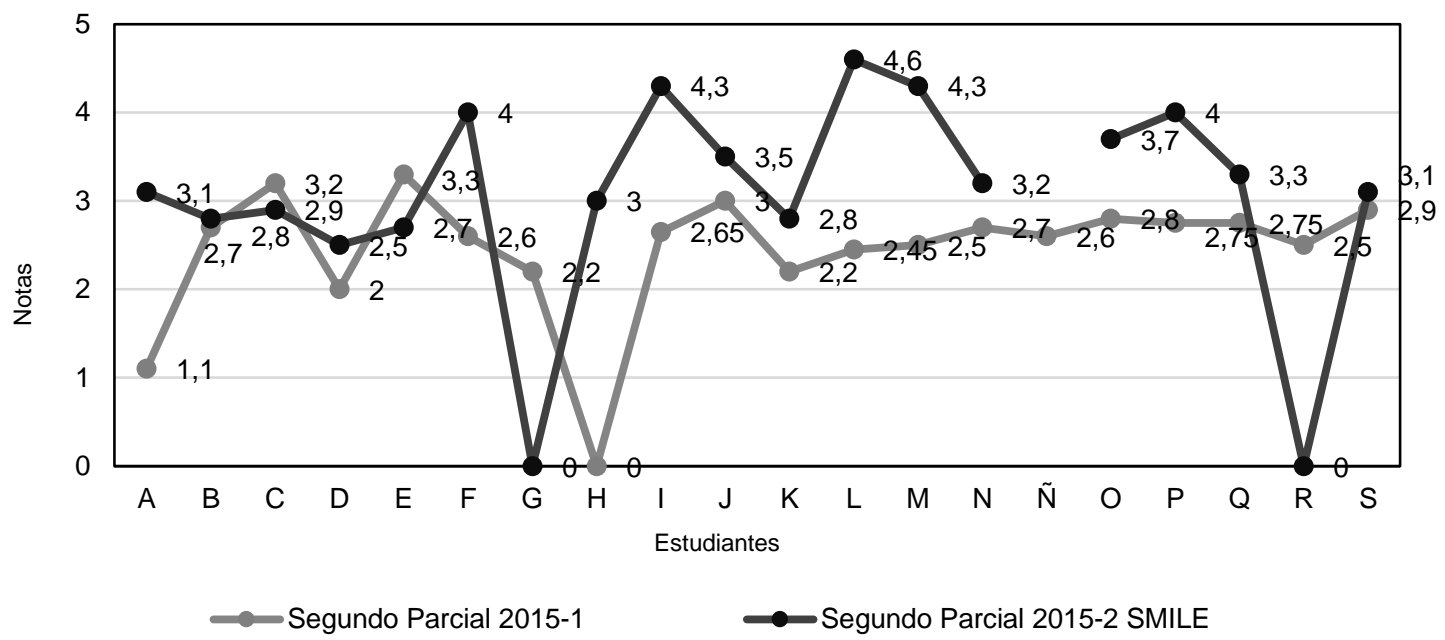

Fig. 2: Resultados Segundos Parciales I y II Sem 2015

La figura 3, muestra los resultados del examen final. Los 2 primeros examenes estan diseñados para evaluar contenidos parciales y el examen final es acumulativo. Todos los examenes se hicieron conservando este criterio y teniendo en cuenta los contenidos vistos. Se usaron preguntas de conocimiento, comprensión y análisis

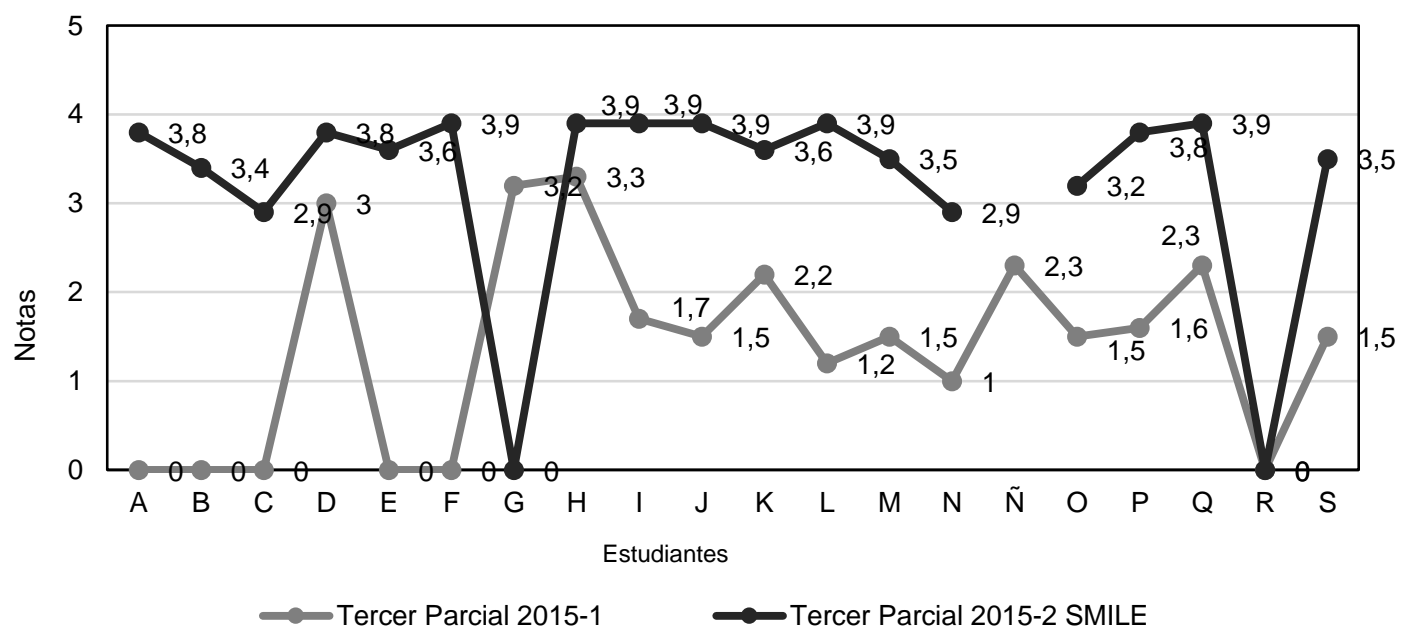

Fig. 3: Resultados por notas y estudiantes del tercer examen I y II Sem 2015 


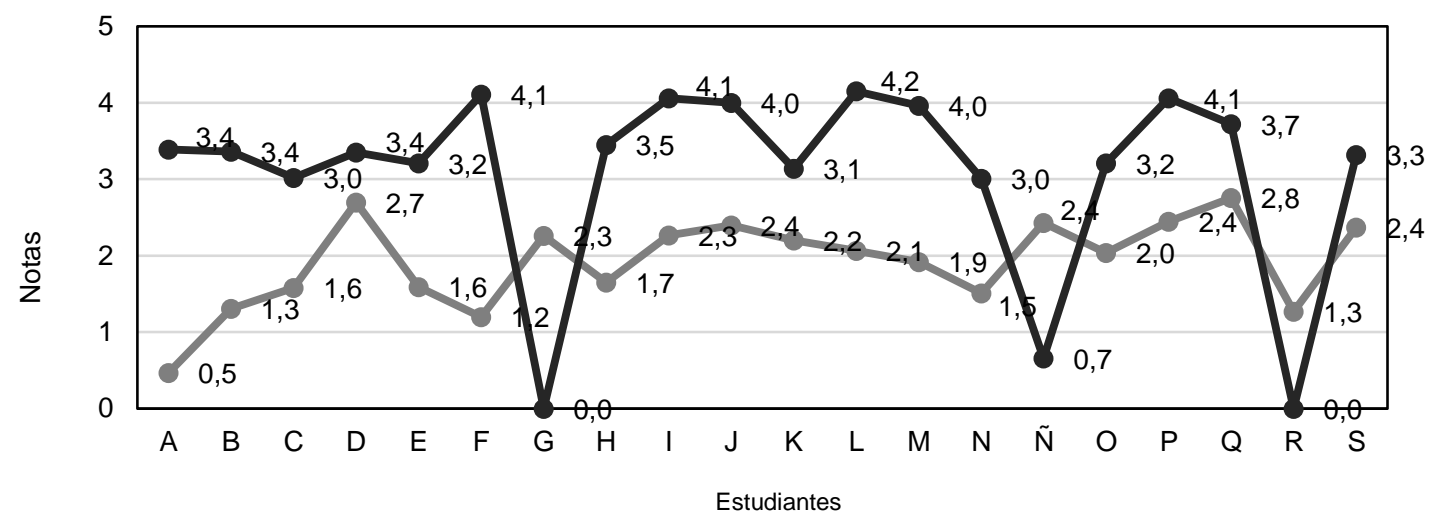

$\longrightarrow$ Nota Definitva 2015-1 $\longrightarrow$ Nota definitiva 2015-2 SMILE

Fig. 4: Resultado final obtenido por los estudiantes I y II Sem. 2015

La figura 4 muestra las notas definitivas con satisfactorios resultados. Las comparaciones se hacen con los resultados de los estudiantes de sus exámenes en el semestre anterior, porque son los mismos contenidos temáticos evaluados, los mismos estudiantes, pero la didáctica es diferente. Lo que es objeto de análisis aquí es valorar cómo a través de esta didáctica se observan cambios notorios, positivos en el aprendizaje de ellos.

Los estudiantes transitaron hacia diferentes fuentes de aprendizaje. Al inicio se apoyaron solo en buscadores de internet y luego fueron haciendo uso de sus notas de clase. La principal fuente fueron los buscadores de internet, pero fue aumentando el uso de sus notas. Se considera que los estudiantes al momento de construir sus preguntas tomaron conciencia de la necesidad de contar con información. Esto, asociado al logro de la competencia de pensar con información que se alcanzó en alto grado. Reconocieron lo importante de atender las sesiones de clase y tomar apuntes concretos sobre lo que consideraban valioso.

La construcción de pensamiento crítico aquí, se centró en la valoración de ellos alrededor de dos aspectos: la evaluación de sus preguntas y respuestas y las preguntas y respuestas hechas por sus compañeros. Tuvieron la capacidad para reconocer preguntas mal o bien escritas, con faltas de ortografía, fáciles o no de comprender, respuestas mal elaboradas, así como preguntas bien escritas, con sentido y bien elaboradas. La plataforma Smile permite que los estudiantes evalúen con estrellas todas las preguntas que van respondiendo y dejen comentarios. En la tabla 3 se presenta el registro de las evaluaciones de los estudiantes.

Tabla 3: Valoración de los estudiantes a las preguntas construidas por sus compañeros en la plataforma Smile

\begin{tabular}{|c|c|c|c|c|c|}
\hline & $\begin{array}{l}\text { 1ra. sesión - } 38 \\
\text { preguntas }\end{array}$ & $\begin{array}{l}\text { 2da. sesión - } 93 \\
\text { preguntas }\end{array}$ & $\begin{array}{l}\text { 3ra. sesión - } 25 \\
\text { preguntas }\end{array}$ & $\begin{array}{l}\text { 4ta. sesión - } 39 \\
\text { preguntas }\end{array}$ & $\begin{array}{l}\text { 5ta. Sesión }-87 \\
\text { preguntas }\end{array}$ \\
\hline $\begin{array}{l}\text { Preguntas bien } \\
\text { elaboradas }\end{array}$ & $26 \%$ & $30 \%$ & $40 \%$ & $56 \%$ & $60 \%$ \\
\hline $\begin{array}{l}\text { Juicios a las } \\
\text { calificadas como } \\
\text { mal elaboradas }\end{array}$ & $\begin{array}{ll}\begin{array}{l}\text { Errores } \\
\text { ortografía, }\end{array} & \text { de } \\
\text { respuestas } & \text { mal } \\
\text { construidas; } & \\
\text { preguntas } & \text { mal } \\
\text { construidas. } & \end{array}$ & $\begin{array}{lr}\text { Errores } & \text { de } \\
\text { ortografía; } & \text { Mal } \\
\text { elaboradas } & \text { las } \\
\text { preguntas y las } \\
\text { respuestas }\end{array}$ & $\begin{array}{lr}\text { Errores } & \text { de } \\
\text { ortografía. } & \\
\text { Opciones que no } \\
\text { aplican para hacer } \\
\text { selección; } \quad \text { mal } \\
\text { construida } & \text { la } \\
\text { pregunta } & \end{array}$ & $\begin{array}{l}\text { Opciones que } \\
\text { no aplican para } \\
\text { seleccionar } \\
\text { respusta; mal } \\
\text { construida la } \\
\text { pregunta; } \\
\text { repetición en las } \\
\text { opciones de } \\
\text { respuesta }\end{array}$ & \begin{tabular}{lr}
\multicolumn{2}{l}{ Opciones que no } \\
aplican para \\
seleccionar la \\
respuesta; mal \\
construida la \\
pregunta; no se \\
comprende la \\
pregunta; No hay \\
suficiente \\
información en la \\
pregunta para \\
responder
\end{tabular} \\
\hline
\end{tabular}

Fueron puntuales en identificar errores de los compañeros y críticos en precisar cuáles eran las razones de ello, afinando cada vez más el nivel de detalle con el que registraban sus juicios. Con respecto a las competencias digitales y el uso de la plataforma Smile, resultó fácil para ellos y motivador el uso y acceso a la plataforma para desarrollar actividades de aprendizaje. Aunque decían que esta no era una clase normal, porque consideraban que "lo normal era que la profesora enseñara los conceptos y diese las respuestas a las preguntas que hacían", - comentario este hecho por ellos en una sesión de clases-, valoraron al final positivamente el proceso de construcción de preguntas como una manera de aprender y la plataforma Smile como mediación tecnológica. 
El uso de la plataforma Smile y su entorno tecnológico, aunque no era el centro de la estrategia de aprendizaje que era la indagación, fue el elemento jalonador para que los estudiantes se desprendieran de las negaciones que tenían por ser repitentes y se dedicaran a realizar las actividades. El dialogo participativo y la observación participante durante las sesiones de clases, permitió reconocer que los estudiantes aunque tenían temor de escribir preguntas y respuestas, les parecía motivador elaborarlas y que sus compañeros las respondieran. Era agradable identificar quien(es) de sus compañeros, habían respondido acertadamente sus preguntas. Se identificó que era motivo de burla entre ellos, las preguntas construidas con errores de ortografía y evaluadas negativamente por parte de todos. Esto a veces les significaba negaciones para querer elaborarlas. En las sesiones finales, estos miedos se fueron dejando de lado y la disposición de todos los estudiantes a escribir las preguntas era total.

\section{CONCLUSIONES}

Se puede concluir que los estudiantes se acercaron a la comprensión de los contenidos de la asignatura de Introducción a la Ingeniería de Sistemas, y superaron las dificultades y debilidades que presentaban en su condición de estudiantes repitentes, usando la Plataforma Smile. 1) Para ellos fue difícil asumir la estrategia de aprendizaje, porque debían combinar la autogestión de información y competencias comunicativas y habilidades de orden superior, elementos estos que no tenían apropiados previamente. Esta combinación les tomo tiempo y esfuerzo cognitivo, pero se aproximaron. 2) Las competencias que más les costó lograr fueron las comunicativas y la de pensar con información, porque debían escribir frases bien elaboradas y construir preguntas con sustento teórico y conceptual, así como proponer respuestas. 3) La competencia de análisis que demandaba ser críticos en la apropiación de temas vistos y convertirlos en preguntas y respuestas, se fue generando y se logró básicamente. 3) Su mayor nivel de pensamiento crítico se logró en la evaluación de las preguntas elaboradas por sus compañeros y en expresar con claridad las razones de sus juicios 4) Las competencias digitales para la incorporación de una plataforma que tenía rutas de acceso, programación y reglas para su uso fue lograda satisfactoriamente. 5) El aporte de la plataforma Smile se centró en el reconocimiento de parte de los estudiantes que era motivador para ellos acceder a ella, elaborar preguntas y que sus compañeros pudiesen responderlas; lo veían como un reto. Los estudiantes se acoplaron a una estrategia de indagación en la que la Plataforma Smile realizó un aporte como motivación extrínseca fácil de comprender y de usar. 6) La taxonomía propuesta del CCA haciendo uso de la estrategia de indagación y el andamiaje haciendo uso de la Plataforma Smile, favorecieron en un alto porcentaje el aprendizaje, generando desarrollo cognitivo en los estudiantes.

\section{REFERENCIAS}

Aaron M., O. Castañeda y A. Ibarra, The Management and Construction of Knowledge as an Innovation Strategy for Collaborative Learning Through the Use and Creation of Learning Communities and Networks, ISSN: 1548-0666, The International Journal of Knowledge Management (IJKM) (2014)

Acosta O., P. Behar y E.B. Reategui, Content recommendation in an inquiry-based learning environment. In Frontiers in Education Conference FIE, 2014, IEEE, 1-6, IEEE (2014)

Arceo F. y G. Rojas, Estrategias docentes para un aprendizaje significativo, Una interpretación constructivista, Editores Mc Graw Hill (2007)

Azevedo, R. y A. Hadwin, Scaffolding self-regulated learning and metacognition-Implications for the design of computerbased scaffolds (2005)

Azevedo, R., J. Cromley y D. Seibert, Does adaptive scaffolding facilitate students' ability to regulate their learning with hypermedia? Contemporary Educational Psychology, 29(3), 344-370 (2004)

Badia A., C. García, Incorporación de las TIC en la enseñanza y el aprendizaje basados en la elaboración colaborativa de proyectos, ISSN: 1698-580X, Revista de Universidad y Sociedad del Conocimiento, 3 (2) (2006)

Barberà, E., Pautas para el análisis de la intervención en entornos de aprendizaje virtual: dimensiones relevantes e instrumentos de evaluación, Universidad Oberta de Cataluña UOC (2004)

Bruner, J., Berlyne Memorial Lecture: Acquiring the uses of language, Canadian Journal of Psychology / Revue canadienne de psychologie, 32(4), 204 (1978)

Buckner, E. y P. Kim, Integrating technology and pedagogy for inquiry-based learning: The Stanford Mobile Inquiry-based Learning Environment (SMILE), Prospects, 44(1), 99-118 (2014)

Frías, M. y A. López, Impacto del ABP en el Desarrollo de la Habilidad para Formular Preguntas de Aprendizaje en Estudiantes Universitarios, REDU: Revista de Docencia Universitaria, 9(1), 57(2011)

Gonzalvez, A., The context: an element of analysis for teaching, Zona Próxima, (25), 34-48 (2016)

Hadwin, A. y P. Winne, CoNoteS2: A software tool for promoting self-regulation educational research and evaluation, Educational Research and Evaluation, 7, 313-334 (2001) 
Hederich Martínez, C., Efecto de un andamiaje para facilitar el aprendizaje autorregulado en ambientes hipermedia, Revista Colombiana de Educación, 0(58) (2012) (2017)

H. Hui-Yin y P. Kim, Preservice teachers' uses of SMILE to enact student-generated questioning practices, International Journal of Innovation in Education, 110-121 (2016)

Huapaya, C.R., G.M., Arona y F.A Lizarralde, Enseñanza de la Ingeniería con Sistemas Tutoriales Inteligentes, https://dx.doi.org/10.4067/S0718-07642005000500012, Información Tecnológica, 16(5), 75-78. (2005)

Kim, P., Action research approach on mobile learning design for the underserved. Educational Technology Research and Development, 57, 415-435 (2009)

Kim, P., T. Miranda y C. Olaciregui, Pocket school: Exploring mobile technology as a sustainable literacy education option for underserved indigenous children in Latin America, International J. of Educational Development, 28(4), 435-445 (2008)

Lee, V., What is inquiry-guided learning? DOI:10.1002/tl.20002, New directions for teaching and learning, 129, 5-14 (2012)

López, V., Efecto de un andamiaje para facilitar el aprendizaje autorregulado en ambientes hipermedia, Revista colombiana de educación, Universidad Pedagógica Nacional, Bogotá, Colombia, 58, 14-39 Colombia (2010)

Maquilón J. y F. Hernández, Influencia de la motivación en el rendimiento académico de los estudiantes de formación profesional, Revista electrónica interuniversitaria de formación del profesorado, 14(1) (2011)

Nonaka, I. y G. Von Krogh, Perspective-Tacit knowledge and knowledge conversion: Controversy and advancement in organizational knowledge creation theory, Organization science, 20(3), 635-652 (2009)

Paris, S.G. y J. Byrnes, The constructivist approach to self-regulation and learning in the classroom. In Self-regulated learning and academic achievement, 169-200, Springer, New York (1989)

Pina, A.B., Blended learning, Conceptos básicos, Pixel-Bit, Revista de medios y educación, (23), 7-20 (2004)

Raes, A., T. Schellens, B. De Wever y E. Vanderhoven, Scaffolding information problem solving in web-based collaborative inquiry learning, Computers \& Education, 59(1), 82-94 (2012)

Reeve, J., Motivación y emoción, 3ª Ed., Mc Graw Hill, México (2003)

Rodríguez, J. y A. Remesal, Estrategias de aprendizaje y rendimiento académico en estudiantes universitarios, Revista de investigación educativa, 25(2), 421-441 (2007)

Seol, S. y P. Kim, Proyecto Stanford Mobile Inquiry-based Learning Environment (SMILE): using mobile phones to promote student inquires in the elementary classroom (2011)

Schunk, D. y B. Zimmerman, Self-regulation of learning and performance: Issues and educational applications, Lawrence Erlbaum Associates, Inc. (1994)

Suárez, Á. y otros cuatros autores, Review of the types of mobile activities in mobile inquiry-based learning, Computers \& Education, 118, 38-55 (2018)

Toro, A. y L. Marcano, Consideraciones teórico epistemológicas en la obra de Vigotski, Revista Ciencias de la Educación, 1(25), 4-61 (2006)

Torrano, F. y M. González, El aprendizaje autorregulado: presente y futuro de la investigación, Electronic Journal of research in educational psychology, 2(1) (2004)

Tornero, B., A. Ramaciotti, A. Truffello y F. Valenzuela, The Cognitive Level of Questions Asked by Preschool Teachers. Educación y Educadores, 18(2), 261-283 (2015)

Turpo, O., Contexto y desarrollo de la modalidad educativa blended learning en el sistema universitario iberoamericano, Revista mexicana de investigación educativa, 15(45), 345-370 (2010)

Vargas, O. y C Martinez, Efecto de un andamiaje para facilitar el aprendizaje autorregulado en ambientes hipermedia, Revista Colombiana de Educación, (58), 14-39 (2010)

Vygotsky, L., Mind in society: The development of higher psychological processes.Cambridge, MA: Harvard University Pres (1978)

Widiana, I. e I. Jampel, Improving Students' Creative Thinking and Achievement through The Implementation of Multiple Intelligence Approach with Mind Mapping. International Journal of Evaluation and Research in Education (IJERE), 5(3), 246-254 (2016) 
